\title{
Griginal Role of morphology and Africle immunohistochemistry in the diagnosis of
cryptic and occult cancer of gall bladder
}

\author{
Manjula Jain, Shivali Sehgal, Anita Nangia, Shilpi \\ Agarwal
}

ABSTRACT

Department of Pathology,

Background: To analyze the role of morphology and immunohistochemistry Lady Hardinge Medical College,

New Delhi-110001.

Correspondence: Shivali Sehgal

Email:shivalisehgal@gmail.com in the diagnosis of cryptic and occult cancers in cases of gall stone disease. Materials and Methods: A total of 125 cases of gall stone disease were analyzed. Three sections were taken from the neck, body and fundus. Additional sections were taken from all grossly abnormal areas. Immunohistochemistry was performed in cases showing atypia on histopathological examination. A panel of p53, CEA and Ki-67 were used.

Results: Gall baldder cancer was detected in $5.7 \%$ of cholecystectomy specimens. The incidence of malignancy was the highest for gall bladders having inflammation with atypia followed by those with inflammation and hyperplasia; and finally inflammation with intestinal atypia. Additional sectioning and immunohistochemistry is mandatory in gall bladders having such changes to rule out an underlying malignancy. Among gall bladders having inflammation with pyloric metaplasia or inflammation alone, no suspicious lesion was noted on additional sectioning.

Conclusion: Careful attention to morphological abnormalities in all cholecystectomy specimens is mandatory. In gall bladders having inflammation with or without pyloric metaplasia, taking additional sections is unnecessary.

KEYWORDS: Gall bladder; morphology; immunohistochemistry; occult; cancer.

\section{Introduction}

Gall stones cause injury to the mucosal columnar epithelium and cause changes like metaplasia, dysplasia and neoplasia. ${ }^{1}$ Mucosal changes like metaplasia and dysplasia are not infrequently found in mucosa close to invasive carcinoma. In addition, atypical hyperplasia may progress to carcinoma in situ and a proportion of such 
cases to invasive carcinoma. ${ }^{2}$ Thus, the presence of such changes in the microscopic sections of cholecystectomy specimens may be pointers to an underlying malignancy. Extensive sampling of the gall bladder and ancillary immunohistochemical panels can help in the diagnosis of such lesions.

The aim of this study was to analyze the role of morphology and immunohistochemistry in the diagnosis of cryptic and occult cancers in cases of gall stone disease.

\section{Materials and Methods}

The study was conducted in Department of Pathology, Lady Hardinge Medical College for a period of 3 months. A total of 125 cases of gall stone disease were analyzed. These were consecutive samples of cholecystectomy specimens received in the histopathology laboratory. Gross examination of the gall bladder was done and type of stone was identified. Three sections were taken from the neck, body and fundus of the gall bladder. Additional sections were taken from all grossly abnormal areas.

On microscopic examination, the lesions were grouped under those having "inflammation only", "inflammation with metaplasia (pyloric/intestinal)", "inflammation with hyperplasia", "inflammation with atypia" and "frank adenocarcinoma". Metaplasia is the replacement of one cell type by another cell type. In the gall bladder, pyloric metaplasia is identified by the presence of tall columnar mucous containing epithelium with flat basal nuclei. Intestinal metaplasia is identified by the presence of tall columnar epithelium with goblet cells. Hyperplasia is defined as increase in the number of cells and in the gall bladder it is identified by pseudostratification of the tall columnar epithelium with nuclear crowding, and occasional mitotic figures. Atypia indicates an increase in the nucleocytoplasmic ratio of the lining epithelial cells, with cells having enlarged nuclei, with irregular nuclear membrane and coarse chromatin. Frequent mitotic activity is seen in lining epithelium with atypia.

Immunohistochemistry was performed in cases showing atypia on histopathological examination. A panel of p53, CEA and Ki-67 were used.
For immunohistochemistry, the sections were deparaffinized and rehydrated with consecutive solution changes of xylene, $100 \%$ ethanol, $95 \%$ ethanol, $80 \%$ ethanol, 70\% ethanol and distilled water. Heat induced epitope retrieval was done by microwave irradiation. The slides were then allowed to cool at room temperature. Next, the sections were incubated with the primary antibody at appropriate dilutions overnight at room temperature. After washing in buffer, the sections were incubated with horse radish peroxidase conjugated secondary antibody at appropriate dilution. After washing in buffer, the slides were developed with 3,3'-diaminobenzidine till the color was appropriate. The slides were then rinsed with tap water, counterstained with hematoxylin and mounted, after consecutive changes in ethanol and xylene.

\section{Results}

One twenty five cases of gall stone disease, all of which were treated by cholecystectomy were included in the study. The age of the patients ranged from 5 to 50 years with maximum cases belonging to the age group of 31-40 years. The male to female ratio was 0.157:1 (17 males and 108 females). All patients presented with symptomatic gall stone disease. Fifty eight percent of the patients had mixed stones, combined stones were found in $26 \%$ of the patients and cholesterol stones in $16 \%$. On gross examination, thickness of the wall of the gall bladder varied from 1-8 $\mathrm{mm}$, the mucosa was velvety or ulcerated except for one case which showed a growth.

Microscopic examination of the 125 cases is discussed in Figure 1. Frank adenocarcinoma was found in the gall bladder which had a growth (Figure 2). Forty seven cases showed inflammation alone. Inflammation with metaplasia was seen in 57 cases; 38 cases had pyloric metaplasia and 19 cases had intestinal metaplasia. Additional sections were taken from all these cases. None of the patients with inflammation alone (47/47) or those with inflammation and pyloric metaplasia showed any evidence of atypia in the additional sections. However, four out of the 19 patients with intestinal metaplasia showed focal atypia on re-sectioning. Immunohistochemistry revealed nuclear positivity for p53, cytoplasmic CEA and high Ki-67 index in these four cases, confirming 
the presence of atypia (Figure 3). Serial sections were taken in all these 4 cases, two of which revealed adenocarcinoma. Thus the incidence of adenocarcinoma amongst cases with inflammation with intestinal metaplasia was $10.5 \%$.

There were 14 cases of inflammation with hyperplasia. On additional sectioning, two of these cases showed atypia which was confirmed on immunohistochemistry (Figure 4) and further serial sections showed origin of adenocarcinoma in both these cases. The incidence of adenocarcinoma in this group was $14.3 \%$.

Six cases showed inflammation with atypia (both on morphology and immunohistochemistry) (Figure 5). Adenocarcinoma was found in two of these cases on additional sectioning, incidence $=33.3 \%$.

\section{Discussion}

Cholelithiasis produces diverse histopathological changes in the gall bladder. Metaplasia, hyperplasia and dysplasia may be pointers to an underlying malignancy Grossly, 10 to $37 \%$ of gall bladder carcinoma cannot be identified with certainty. ${ }^{3}$ The term "Incidental gall bladder carcinoma" has been defined as, carcinoma of the gall bladder which is not suspected before or at operation and even on gross examination of the opened gall bladder specimen by the surgeon, but is detected for the first time on histopathological examination of a gall bladder removed for presumed (clinical, ultrasound, operative) diagnosis of gall stone disease. Therefore, all gall bladder carcinomas not detected preoperatively and diagnosed during or following surgery are considered as incidental gall bladder carcinomas. ${ }^{4}$ Even on histopathology, malignancy may be focal or cryptic, hiding under benign changes (metaplasia, hyperplasia and dysplasia). Incidence of unsuspected malignancies varied from 0.73 to $2.2 \%$ in past series 5-10. In a recent review, the incidental identification of gall bladder cancer was reported in 0.2 to $3 \%$ of all cholecystectomies done for presumed benign diseases. ${ }^{11}$ One Indian study has reported an incidence of $6.8 \%$

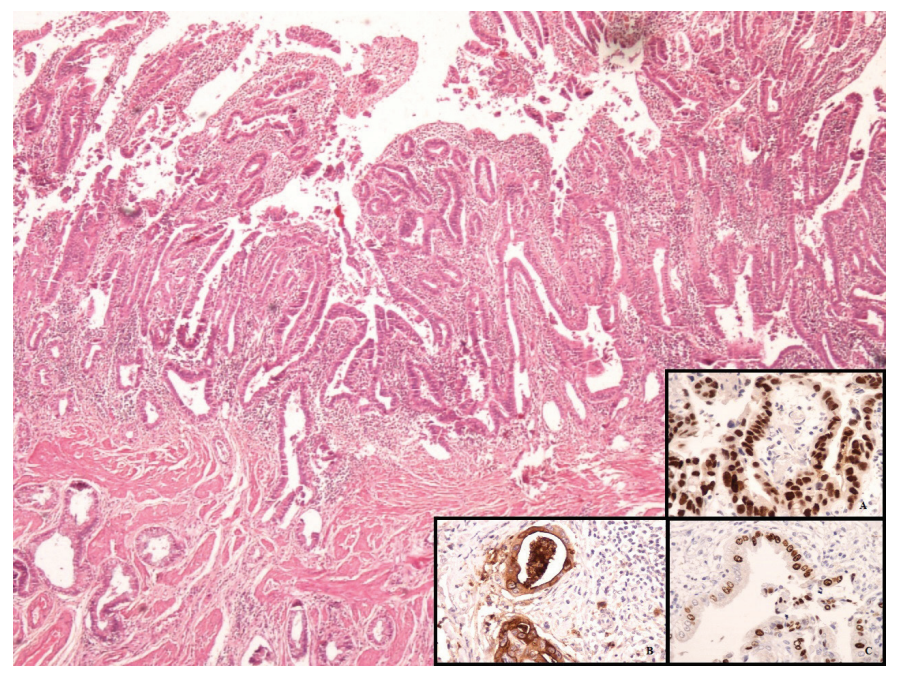

Figure 2: Frank adenocarcinoma in gall bladder showing a growth [HE, 40X] Inset A shows p53 nuclear positivity [400X], inset $B$ shows cytoplasmic positivity for CEA [400X] and inset $\mathrm{C}$ shows $\mathrm{Ki}$ 67 index $=60 \%[400 X]$.

among 415 cholecystectomies studied. ${ }^{12}$ In the current study, the incidence was found to be $5.6 \%$.

Presence of pyloric and intestinal metaplasia is confirmed by presence of respective type of lining epithelia in the gall bladder. Goblet cells are mandatory for labeling intestinal metaplasia. In hyperplasia, there is pseudostratification of the epithelium lined by tall columnar cells with nuclear crowding and presence of occasional mitotic figures. Dysplasia shows epithelial pseudostratification with some loss of architecture, disorganization of the epithelium, nuclear crowding and atypia. ${ }^{13}$ Morphologically, it may be difficult to pick up 


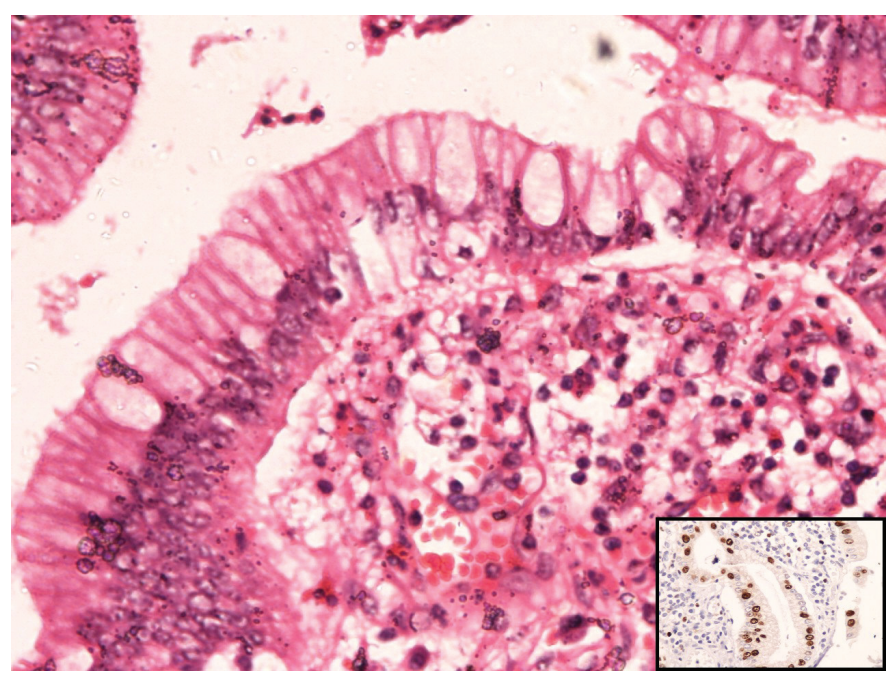

Figure 3: Lining epithelium showing intestinal metaplasia with atypia $[\mathrm{HE}, 400 \mathrm{X}]$ with nuclear positivity for $\mathrm{p} 53$ [inset, $400 \mathrm{X}$ ].

well differentiated adenocarcinoma of the gall bladder. Rockitansky - Aschoff sinuses (RAS) may be located throughout the gall bladder wall, even extending into the perimuscular tissue. RAS are normally continuous, showing a perpendicular orientation to the surface and typically have undulating, smooth contours. Adenocarcinomas, on the other hand, are composed of small and variably sized glands with angulated contours. They are densely packed and arranged parallel to the surface. Presence of desmoplasia favours carcinoma. Cytological atypia, mitoses and necrosis (intraglandular) are features favouring a diagnosis of adenocarcinoma. ${ }^{10,14,15}$

Nuclear positivity for p53, high Ki-67 index and cytoplasmic positivity for CEA is highly suggestive of a typical epithelium of the gall bladder. Nuclear p53 expression is seen in gall bladder epithelium with atypia where it is present in more than $30 \%$ of all dysplasia in the extrahepatic biliary tract.The incidence and degree of expression of p53 is significantly higher in high grade lesions, while relatively uncommon in non neoplastic epithelium. However, this expression can also be seen in areas of marked regenerative changes, which limits its use as a sole diagnostic marker. ${ }^{16}$ The Ki-67 labelling index is higher in areas of gall bladder showing atypia. It is greater in dysplastic lesions and increases in quantity with increasing degrees of dysplasia, however, it can also be seen in areas of regenerative change. ${ }^{16}$ CEA which

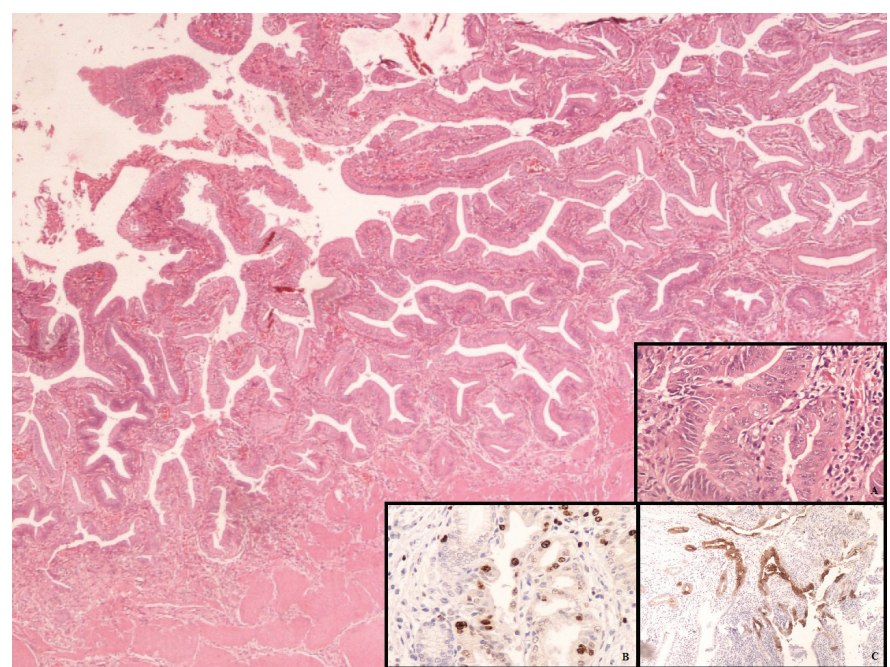

Figure 4: Hyperplasia of the epithelium [HE, 40X] with inset A showing atypia of the cells [HE,400X] Inset $B$ shows $\mathrm{p} 53$ nuclear positivity [400X] and inset $B$ showing CEA cytoplasmic positivity [40X].

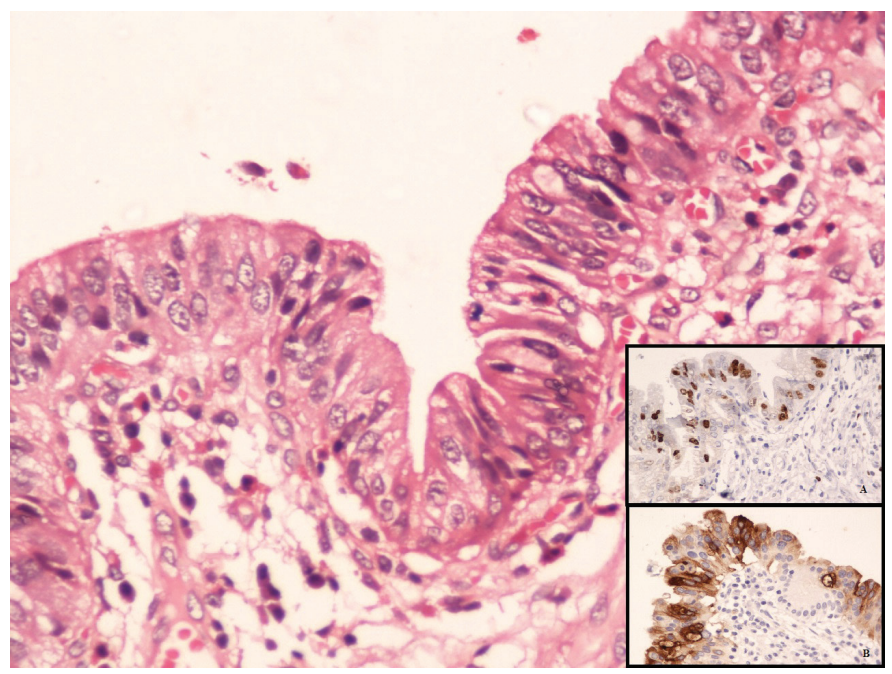

Figure 5: Lining epithelium showing atypia [HE, 400X] with inset $A$ showing 153 nuclear positivity [400X] and inset B showing CEA cytoplasmic positivity [400X].

is normally limited to the apical membrane of benign cells, are commonly detected in the cytoplasm of the adenocarcinoma cells. There is a progressive increase in the level of expression from pre-invasive to invasive and to poorly differentiated carcinomas with dense intracytoplasmic expression detected mostly in advanced carcinomas. ${ }^{16}$ Presence of cytoplasmic expression of CEA 
is a pointer to the presence of atypia. While each of these markers per se may not be useful for detection of atypia, a panel of all 3 almost confirms the presence or absence of atypia in the gall bladder.

Khanna et al found that epithelial hyperplasia was the most frequent morphological change seen in $60 \%$ of the patients with gall stone disease (140 cases). ${ }^{13}$ In our study, metaplasia was the most common change followed by hyperplasia and atypia.

The malignant potential of metaplasia, hyperplasia and atypia is stated to be uncertain, but probability of malignant transformation would progressively increase from hyperplasia to metaplasia to dysplasia. ${ }^{13}$ In the current study, the incidence of malignancy was the highest for gall bladders having inflammation with atypia followed by those with inflammation and hyperplasia and finally those with inflammation with intestinal metaplasia. Additional sectioning and immunohistochemistry is mandatory in gall bladders having such changes to rule out an underlying malignancy. Among gall bladders having inflammation with pyloric metaplasia or inflammation alone, no suspicious lesion was noted on additional sectioning. Therefore, in gall bladders having inflammation with or without pyloric metaplasia, taking additional sections is unnecessary.

It is important to note that though the prognosis of gall bladder carcinomas is poor, incidental gall bladder carcinomas have better outcomes. ${ }^{17,18}$ This is due to the fact that tumors detected on histopathology usually present early stage disease and patients get promptly referred for definitive surgery. Completion radical cholecystectomy is the standard treatment for incidental gall bladder carcinomas that are stage I $b$ and beyond. ${ }^{17,19,20}$

\section{Conclusion}

Careful attention to morphological abnormalities in all cholecystectomy specimens is mandatory. This study provides basic knowledge of the spectrum of pathological findings in cholelithiasis. Microscopic examination facilitates detection of premalignant and malignant lesions which are not apparent even on gross examination of the specimen. Immunohistochemistry is an important tool aiding in the identification of cryptic malignancies of the gall bladder.

\section{References}

1. Gupta SC, Misra V, Singh PA, Roy A, Misra SP, Gupta AK. Gall stones and carcinoma gall bladder. Indian J Pathol Microbiol. 2000;43(1):147-54.

2. Kelly P. Gallbladder and biliary tract. McGee J O'D, Isaacson PG, Wright NA (ed). Oxford university press, New York. 1992. pp 1401-17.

3. Roa I, Araya JC, Villaseca M, Roa J, deArtxabale X, Ibacache G. Gall bladder cancer in a high risk area: morphological features and spread pattern. Hepatogastroenterology. 1999;46:1540-6.

4. AgarwalAK, Kalayarasan R, Sakhuja P.Allcholecystectomy specimens must be sent for histopathology to detect inapparent gall bladder cancer. HPB. 2012;14:269-73.

5. Bani-Hani KE, Yaglan RJ, Matalka II, Shantnawi NJ. Gall bladder cancer in Northern Jordan. $J$ Gastroenterol Hepatol. 2003;18(8):954-9.

6. Gurleyik G, Gurleyik E, Ozturk A, Unamiser S. Gall bladder carcinoma associated with gall stones. Acta Chir Belg. 2002;102:203-6.

7. Mohan H, Punia RD, Dhawan SB, Ahal S, Sekhon MS. Morphological spectrum of gall stone diseases in 1100 cholecystectomies in North India. Indian J Surg. 2005;67:140-2.

8. Zahrani IH, Mansoor I. Gall bladder pathologies and cholelithiasis. Saudi Med J. 2001;22(10):885-9.

9. Kaur A, Dubey VK, Mehta KS. Gall bladder mucosal changes associated with chronic cholecystitis and their relationship with carcinoma gall bladder. JK Science. 2012;14(2):89-92.

10. Terada T. Histopathologic features and frequency of gall bladder lesions in consecutive 540 cholecystectomies. Int $J$ Clin Exp Pathol. 2013;6(1):91-6.

11. Kanthan R, Senger JL, Ahmed S, Kanthan SC. Gall bladder cancer in the 21st century. $J$ Oncol. 2015;2015:967472.

12. Tyagi SP, Tyagi N, Maheshwari V, Ashraf SM, Sahoo P. Morphological changes in diseased gall bladder: a study of 415 cholecystectomies at Aligarh. J Indian Med Assoc. 1992;90(7):178-81.

13. Khanna R, Chansuria R, Kumar M, Shukla HS. Histological changes in gall bladder due to stone disease. Indian J Surg. 2006;68(4):201-4.

14. Albores-Saavedra J, Shukla D, Carrick K, Henson DE. 
In situ and invasive adenocarcinomas of the gall bladder extending into or arising from Rockitansky-Aschoff sinuses: a clinicopathological study of 49 cases. Am J Surg Pathol. 2004;28(5):621-8.

15. Saul SH. Gall bladder and extrahepatic biliary tree. In Sternberg's Diagnostic Surgical Pathology. Volume Chapter 35. 5th edition. $L W W$. 2009:1205-27.

16. Dabbs DJ. Diagnostic Immunohistochemistry. 4th ed. Philadephia (PA): Elsevier Saunders;2014.

17. Lai CH, Lau WY. Gall bladder cancer - a comprehensive review. Surgeon. 2008;6:101-10.

18. Kaushik SP. Current perspectives in gall bladder carcinoma. J Gastroenterol Hepatol. 2001;16:848-54.

19. You DD, Lee HG, Paik KY, Heo JS, Choi SH, Choi DW. What is an adequate extent of resection for T1 gall bladder cancers? Ann Surg. 2008;247:835-38.

20. Wakai T, Shirai Y, Hatakeyama K. Radical second resection provides survival benefit for patients with T2 gall bladder carcinoma first discovered after laparoscopic cholecystectomy. World J Surg. 2002;26:867-7. 